\section{Cahiers de Narratologie}

Analyse et théorie narratives

$10.1 \mid 2001$

La voix narrative

\title{
Le viol du silence et la parole exacerbée : Chronique de la maison assassinée de Lucio Cardoso
}

Jacqueline Bernard

\section{(2) OpenEdition}

1 Journals

\section{Electronic version}

URL: http://journals.openedition.org/narratologie/6929

DOI: 10.4000/narratologie.6929

ISSN: 1765-307X

\section{Publisher}

LIRCES

\section{Printed version}

Date of publication: 1 January 2001

Number of pages: 137-147

ISBN: 2914561032

ISSN: 0993-8516

\section{Electronic reference}

Jacqueline Bernard, "Le viol du silence et la parole exacerbée : Chronique de la maison assassinée de Lucio Cardoso", Cahiers de Narratologie [Online], 10.1 | 2001, Online since 20 October 2014, connection on 24 February 2021. URL: http://journals.openedition.org/narratologie/6929 ; DOI: https://doi.org/ 10.4000 /narratologie.6929 


\title{
LE VIOL DU SILENCE ET LA PAROLE EXACERBÉE : CHRONIQUE DE LA MAISON ASSASSINÉE DE LUCIO CARDOSO
}

\author{
Jacqueline BERNARD \\ Université Stendhal, Grenoble III
}

La Chronique de la maison assassinée ${ }^{1}$, de Lucio Cardoso, roman unique dans la littérature brésilienne, paru en 1959, possède une structure qui évoque naturellement Choderlos de Laclos, Gide ou Dostoïevski. Le roman est un texte éclaté en discours particuliers, une sorte de puzzle énonciatif, système qui crée le code d'écriture et de lecture de l'ensemble.

Pour qui ne connaîtrait pas ce roman, je vais très rapidement évoquer la trame de la fiction : une jeune femme de Rio, Nina, épouse Valdo, l'un des frères du clan Meneses, propriétaires fonciers dans une petite ville du Minas Gerais. L'incompréhension est totale entre la vie que menait Nina, la carioca, et les habitants du Domaine, avec un grand « D ", endormis dans la rigide torpeur des coutumes et anéantis par la dégradation économique, ce qui les oblige à des compromis incessants entre l'être et le paraître. L'hostilité du frère aîné, Demetrio, qui ne peut accepter aucun changement et de sa femme Ana qui est victime de son éducation puritaine, rendent le séjour de Nina impossible, même avec l'aide neutre mais indulgente de la gouvernante Betty ou avec le dialogue qu'elle sait engager avec le troisième frère, Timoteo, personnage extravagant considéré comme fou.

${ }^{1}$ Lucio CARDoso, Crônica da casa assassinada, Editora Nova Fronteira, Rio de Janeiro, 1959. Traduction française de Mario Carelli, Chronique de la maison assassinée, Éd. A. M. Métailié et Eds Mazarine, Paris, 1985. 
J'ai retenu ce roman à cause de son architecture narrative et des occurrences de l'énonciation qui forgent la cohérence d'un récit, présenté en éclats. En effet, le roman est exclusivement constitué d'une suite de textes de types divers (fragments de journal, lettres, confession ou mémoire...) rédigées par les différents personnages sans que ces écrits soient jamais surplombés, semble-t-il, par quelque intervention d'un auteur-narrateur ou commentateur. Partant de là, les deux questions que je me propose d'examiner sont :

- Y a-t-il, au-delà des différents documents produits par les personnages-rédacteurs, une unité énonciative, ou, au contraire un dialogisme irréductible?

- Quelles sont les qualités performatives des différents discours ? Il s'agira d'évaluer les choix linguistiques attribués aux différents destinateurs ainsi que l'articulation entre les destinataires intra-textuels (les personnages) et le destinataire extra-textuel (le lecteur)?

\section{Fragmentation énonciative}

Pour apporter des réponses à cette première question, je suivrai deux axes d'observation : l'organigramme des textes et les marqueurs génériques.

\section{A. L'agencement des textes}

56 textes sont offerts à la lecture, produits par 10 rédacteurs qui ont, d'une manière ou d'une autre, été impliqués dans un drame. On pense, évidemment, et à juste raison, à une structure de roman à énigme, ce que les derniers textes, et surtout l'ultime, confirment en levant l'hypothèque sur une ambiguïté soigneusement entretenue. J'ajouterai qu'il existe, en réalité 11 actants mais l'un deux, Demetrio, tout en étant très important, est muet. Il n'existe que par la parole rapportée des autres. Chacun des rédacteurs propose plusieurs interventions et leurs textes, fragmentés, se présentent assez souvent comme des entités qui peuvent fonctionner d'une manière à peu près autonome. En outre, si le retour des rédacteurs n'est pas géré par une stricte alternance rythmique, il est notable qu'il y a une structure d'ouverture et de fermeture. Les cinq premiers textes appartiennent à cinq énoncia- 
teurs différents - comme autant d'ouvertures de parenthèses que je qualifierais de rouselliennes, - qui, ensuite, reviendront d'une manière plus aléatoire. A partir du chapitre 42 , les titres referment progressivement ces parenthèses en mentionnant par exemple : «Dernier récit du pharmacien» ou « Dernière confession d'Ana ».

Ces textes relèvent de quatre genres, nettement marqués à la fois par leurs titres et par les marques énonciatives. En voici le détail selon leur degré décroissant d'intégration à la fiction : la présence de Nina au domaine et les troubles qu'elle engendre : jalousie, rupture du silence ancestral, adultère, inceste.

- Deux personnages rédigent un journal : André, fils présumé de Nina, (11 occurrences) et Betty la gouvernante anglaise (5 occurrences).

- Deux personnages rédigent des lettres: Valdo Meneses ( 2 occurrences) et sa femme Nina (5 occurrences).

- Un personnage rédige une confession: Ana, bellesœur de Nina (10 occurrences).

- Un personnage rédige des mémoires : Timoteo, frère cadet de Valdo (2 occurrences).

- Quatre personnages rédigent un acte testimonial : le pharmacien ( 5 occurrences), le médecin (3 occurrences), le prêtre (5 occurrences) et un colonel ami de Nina qui vit à Rio (1 occurrence).

- Le dernier chapitre doit être mis à part car il ne relève d'aucun des genres précédents. Il s'intitule : "Postscriptum d'une lettre du père Justino ». Nous verrons sa fonction plus tard.

Ces données brutes demandent quelques commentaires pour être interprétables. Tout d'abord, les occurrences signalées sont identifiées par les changements de chapitre mais certaines de ces rédactions sont fragmentées, occupant plusieurs chapitres ou interrompues par d'autres rédactions. En outre, un rédacteur de lettre, Valdo, change une fois de genre de discours pour entrer dans le testimonial, changement qui, on va le voir, est une ressource importante de la narration. 
Les indications ainsi données par le dispositif textuel, ce que j'appellerai l'organigramme du roman, servent d'indices pour évaluer la prééminence des personnages. Il y a ceux qui constituent par leurs actes le drame, ce qui ne les empêche pas de le commenter, et ceux qui commentent de l'extérieur le drame, ce qui ne les empêche pas d'y participer. La durée du discours accordé à chacun détermine indiscutablement son importance. Si une première lecture sans repérage donne immédiatement la première place diégétique à Nina, belle, jeune, étrangère, maîtresse du Domaine par son mariage avec Valdo Meneses, qui assure la continuité thématique, l'observation des rédactions montre que l'énonciateur principal est André et que le personnage nommé Ana arrive en deuxième position. L'organigramme constitué métatextualise la fiction : ainsi y a-t-il un changement de statut du discours tenu par Valdo. Les deux lettres adressées à sa femme, Nina, font place à des interventions qualifiées de "déposition", puis de " témoignages ». Quand il pratique le genre épistolaire, il est constituant à part entière de la fiction; quand il pratique le genre testimonial, il sort de la sphère d'action pour entrer dans une sphère d'observation et de réflexion, au même niveau que les spectateurs, tels que le médecin, le pharmacien ou le prêtre. Dans les premiers cas - les lettres - l'énonciation a un destinateur identifié :

«Je me souviens soudain, tout particulièrement, de cette nuit où tu es venue à mon chevet, pour me dire adieu. Comme je t'aimais à ce moment précis, Nina, quel trouble et quelle indicible douleur ta présence provoquait en moi ! [...] Ah ! je me souviens bien du silence de cette minute : tu ne peux pas t'imaginer combien je me suis senti inexistant et malheureux. » (p. 97)

Dans les autres cas, l'énonciation n'a pas destinataire identifié. Il s'agit, soit du texte qui est nommé « déposition » au chapitre 37 :

« Depuis longtemps nos relations étaient simplement cordiales. J'avais cessé de chercher à la comprendre, convaincu qu'il n'y avait pas d'explication rationnelle à son comportement. J'étais indifférent à ce qu'elle pouvait bien faire, même si parfois je me surprenais à l'observer ou à essayer de deviner la raison de tel ou tel de ses 
gestes, maintenant je voulais seulement qu'il n'y ait pas conflit entre nous. » (p. 283)

soit des textes qui sont nommés "témoignages » dans les chapitres 44 et 47 . Une rupture dans l'emploi des temps, passage brusque de l'imparfait au conditionnel, met en relief la modification du statut de l'énonciateur qui s'expose comme omniscient. Cette variation est d'autant plus significative qu'elle constitue l'unique prolepse du roman :

« En marchant dans l'obscurité, j'ai vu la maison éclairée, des fenêtres ouvertes, une ombre ou deux en train de passer dans les couloirs ; le Domaine, toujours plongé dans le calme, devenait différent pour qui connaissait ses coutumes (p. 338) [...]

Plus tard quand je serais en train d'attendre la voiture pour quitter définitivement le Domaine, Ana viendrait me trouver. Sur la route écrasée par le soleil qui se coucherait déjà du côté de la serra du Bau, elle paraîtrait plus petite comme rétrécie, ses gestes seraient plus timides. » (p. 375)

Valdo, acteur, puis témoin, est donné comme un personnage faible qui subit la loi de son frère, Demetrio, et celle des tabous socio-religieux, qui s'est toujours soumis aux contraintes ancestrales sans rien comprendre du monde, et surtout pas sa femme, une énigme pour lui. Or, le changement de son statut énonciatif le fait passer de la non-connaissance à la connaissance, de la lecture aveugle de son environnement à une lecture plus experte qui lui fait désigner son frère, celui qui n'écrit aucun texte, le blanc indicible, comme le responsable du drame :

«Pour ça tu as menti, tu as trompé et tu as trahi. Tu n'as jamais pu la supporter [...] Possédé par l'esprit maléfique des Meneses, tu n'as jamais pensé à autre chose qu'à détruire cette femme. » (p. 380)

\section{B. Les marqueurs génériques}

Tous les rédacteurs disent "je ». C'est le système dominant du roman. Mais tous ces « je » sont loin d'avoir le même poids, ni la même valeur énonciative. Certains peuvent aisément être remplacés par la troisième personne et c'est en 
réalité comme cela qu'on les lit. Cependant chacune des voix qui intervient se positionne par rapport à « Elle », objet et sujet des discours, selon le degré de sympathie ou de proximité que les rédacteurs ont eu avec Nina.

Les prestations d'André et de Betty, rédacteurs de journaux intimes, sont à rapprocher. Betty, par sa fonction de gouvernante est plutôt dans le montrer: son discours comporte nombre de passages textuels qui se caractérisent par une visualisation importante et une abondance de détails comme ce portrait du plus jeune des frères Meneses :

"Une fois encore j'ai pu constater la bizarrerie des habitudes qui constituaient les lois plus ou moins constantes de son monde [...] j'ai vérifié que monsieur Timoteo, obèse et en sueur, portait une robe à franges et paillettes qui avait appartenu à sa mère. Le corsage était beaucoup trop serré à la ceinture et par endroits les coutures craquaient, laissant apparaître la chair emprisonnée qui déchirait le tissu. » (p. 36)

André, à cause de sa jeunesse, de son éducation et, bien sûr, de sa relation intime et secrète avec Nina est plutôt dans le raconter, bien que son discours résume et commente la diégèse en dérivant vers des réflexions morales ou philosophiques :

«...Mon Dieu, qu'est-ce que la mort? Puisqu'elle s'est éloignée de moi, que la terre abritera bientôt sa dépouille mortelle, combien de temps devrai-je refaire en ce monde le chemin de son enseignement, de son admirable leçon d'amour ? [...] Qu'est-ce que ce pour toujours sinon l'écoulement continu de l'existence, de ce qui est libéré de toute contingence et qui se transforme, évolue et débouche sans arrêt sur des sensations mouvantes?»(p. 7)

Les contributions des autres rédacteurs viennent se caler sur cette matrice énonciative. Les observateurs extérieurs, les rédacteurs des témoignages, pharmacien, médecin, prêtre, tiennent un discours de commentaire sur des morceaux de l'histoire à laquelle ils participent ponctuellement. La distance que souligne le pharmacien est caractéristique : 
« Je m'appelle Aurelio dos Santos, et il y a très longtemps que j'ai ouvert dans notre petite ville ce commerce de médicaments et de drogues pharmaceutiques. [...] Les Meneses, par orgueil et par suffisance étaient les seuls clients qui ne venaient pas chez moi. Ils envoyaient des messages, me faisaient parvenir les ordonnances et payaient par l'intermédiaire de leurs domestiques. [ ...] Je me disais à moi-même : « Ce sont les gens du Domaine ». » (p. 29)

C'est l'un des cas de cette fiction où la substitution de l'énoncé à la troisième personne est envisageable. Cette virtualité situe habilement le personnage en position de regard extérieur.

Par contre, les longues confessions d'Ana et celles, plus courtes de Timoteo, sont des discours impossibles à transformer car les marques de la subjectivité sont très abondantes :

«Si j’écris ces mémoires, mon intention n'est pas de me justifier, - contrairement à ce que beaucoup pourront penser -, ni même de paraître meilleure après ma mort. Non, vraiment pas, c'est d'autant plus vrai que si quelqu'un venait à me lire, ce que l'on penserait à mon sujet ne changerait rien du tout car je ne serai plus que poussière, et peut-être que plus personne ne se souviendra de celle qui aujourd'hui se penche sur cette feuille. » (p. 126)

Tirons maintenant les conséquences des analyses qui précèdent et considérons la Chronique de la maison assassinée comme un espace intertextuel dans lequel se confrontent des discours, ce qui exclut l'idée d'un bloc idéologique où la vérité aurait un référent unique. Nous avons plutôt affaire à un inter-dit, selon le terme de Julia Kristeva ${ }^{2}$. opposant au « monologisme du discours littéraire représentatif [...] une écriture kaléidoscopique et plurielle », inter-dit qui fragmente un je constitué : pas de sujet fixe, pas de destinataire fixe pour l'entendre, pas de sens fixe, pas d'instance auctoriale suprême

2 Préface de Julia Kristeva à Mikhaïl Bakhtine, La poétique de Dostoïevski, op. cit., p. 21. 
qui assurerait la vérité de la confrontation. C'est pourquoi il nous reste à examiner, à travers la performance des discours, la fonction « narrateur » et la place du destinataire.

\section{La performance des discours}

Ce qui fait l'intérêt poétique de l'œuvre de Cardoso est la théâtralisation de l'engendrement du discours de chacun des protagonistes dans le cours même de leur écriture.

\section{- La fonction « narrateur »}

La maison détruite est, en fait, le signe que la parole n'est plus assurée et que la lecture des événements n'a plus de grille normée. La théâtralisation de la personne chez Cardoso est un exemple très bakhtinien de l'autonomie illocutoire du personnage qui offre à la fois une liberté et un inachèvement. Il y a effectivement dans son roman cette structure de dialogisation complète de tous les éléments structuraux qui crée une tension, voire une exaspération de la parole. Le narrateur, quelle que soit son identité (Valdo, le pharmacien, Nina ou Ana) est dans le présent du processus énonciatif. C'est ainsi que peut s'expliquer le choix poétique de l'auteur, ce mélange des prises de paroles qui gomme toute logique chrono-spatiale. Le temps de chaque intervention est le temps d'écriture de l'énonciateur au moment de son écriture et aucune des interventions n'est une délégation de parole d'auteur. La décontextualisation, qui est un fondement de l'écriture littéraire, participe explicitement au choix d'éclatement des énoncés, multipliant les possibilités de connexion.

Ainsi, l'unité monologique est rompue par la « conscience de soi » des intervenants dont la parole est en dialogue constant, faisant coïncider la dominante du « représentant» avec la dominante du «représenté ». La vérité d'Ana est placée en face de celle de Nina, celle du fils, André, est parallèle à celle du père, Valdo. Les événements sont présentés simultanément mais sans hiérarchie valorisante ou dévalorisante, chaque participant défendant librement sa cause et ses choix. Par contre, l'interpénétration des voix dans ce roman polyphonique construit les conditions des multiples interprétations que renferme une opinion donnée. 
Le croisement des points de vue annule toute tentative de vérité ou de message monologique. Il n'a pas d'excédent interprétatif de la part de l'auteur, organisateur des dialogues : il fait se rencontrer personnages et opinions.

Le drame du Domaine qui meurt, métaphorisé autant par le cancer de Nina que par la perversion des conduites tenues secrètes (homosexualité mal assumée de Timoteo, désir refoulé d'Ana qui l'a conduite à la trahison et à la manipulation des autres) n'est jamais l'objet d'un jugement extérieur. La mise en dialogue, direct souvent (échanges d'opinions à l'intérieur de la fiction) ou indirect (lectures successives des documents), trace les contours et les spécificités d'un monde en mutation dont les soubresauts sont matérialisés par les accidents de parcours des personnages qui les analysent avec leurs possibilités au moment où ils parlent. Mais ce présent de l'aveu crée une liaison entre un passé qui est du déjà-là et un avenir qui ne peut pas encore se dire.

Il nous reste à voir maintenant le système de réception qui génère la place que se réserve l'auteur dans ce système.

\section{- La place du destinataire}

Nous avons vu que l'anachronie est le système de dramatisation du roman de Cardoso, sa marque polyphonique. Mais une question se pose tout de même : à qui s'adressent les textes écrits qui se succèdent ? Autrement dit, quelle est la nature du contrat littéraire que propose le roman de Cardoso ?

Le principe actif et explicite est le dialogue, mais quels en sont en réalité les interlocuteurs ? Certains documents ne posent pas trop de problèmes, comme les lettres qui, par essence, supposent un émetteur et un récepteur repérables. Cette facilité d'identification est reconnue à plusieurs reprises avec des titres tels que : Lettre de Nina à Valdo (chapitre 2) ou Lettre de Valdo à Nina (chapitre 10) ou Lettre de Nina au Colonel (chapitres 18 et 35). On voit assez nettement la fonction narrative de ces lettres : elles constituent le moteur diégétique, mettant en relation les personnages principaux en donnant le substrat du roman. Après cela la question se pose : qui est chargé de lire les documents annexes, paroles du 
pharmacien ou du médecin ou encore la confession d'Ana ou le journal de Betty ? Aucun destinataire n'est identifié. Les journaux d'André et de Betty, paroles intimes ont pour finalité de rester au secret. Les témoignages ne sont, en général, requis que ponctuellement, pour un complément d'information.

La dispersion informative du roman de Cardoso distend le lien auteur-lecteur au point que l'effet de texte produit est une forme d'anonymat, poétiquement construit. Le contrat littéraire apparaît donc comme un lien direct entre les textes et le lecteur. Mais cela ne veut pas dire pour autant que l'auteur est absent. Son activité, elle aussi, relève du dialogisme généralisé. Si l'auteur a dans ce texte la place «fantastique » que Bakhtine attribuait à Dostoïevki, on peut la lire dans des lieux de commentaire, les uns explicites, les autres implicites.

Certains textes comportent des mentions qui les surchargent, qui transforment quelque peu le sens attendu. Ainsi la lettre de Valdo à Nina, au chapitre 10 commence par l'indication en italique "Post-scriptum dans la marge de la feuille de papier " suivi de considérations qui auraient pu prendre leur place à l'intérieur de la lettre. Le deuxième fragment du journal d'André au chapitre 17 comporte une indication avec ouverture d'un seul crochet: "[Ajouté avec une autre écriture en marge du cahier: Je ne pourrai jamais oublier la robe qu'elle portait ce soir-là....". Même phénomène au chapitre $26:$ le journal d'André comporte à nouveau une variante du procédé. Entre deux crochets on lit : " [Écrit en marge du cahier, d'une écriture différente : Je ne connaissais pas les femmes. Mais Nina m'a fait comprendre rapidement ce qu'elles sont.] ». Même dispositif dans un passage du journal de Betty, au chapitre quatre quand elle voit Nina pour la première fois: "[Note dans la marge du manuscrit : Aujourd'hui, tant d'années après, rien ne m'a autant impressionnée que cette vision...] ».

Dans tous ces cas, la continuité du discours ne serait pas affectée par l'absence de ces annotations. C'est dire qu'elles ont une fonction particulière qui n'ajoute rien à la diégèse mais révèle la présence d'un auteur-éditeur, procédé qui s'apparente à l'effet de réel selon Barthes. Donc, parallèlement au narrataire ou au lecteur inconnu qui pourraient 
prendre connaissance de ces documents en un temps et en un lieu indéterminés, l'auteur se place en position de lecteur et de commentateur, tel ce sténographe, idéal enregistreur dont parle Bakhtine à propos de Dostoïevski.

Le dernier chapitre, intitulé Post-scriptum du Père Justino est un des lieux possibles de la voix de l'auteur. D'une part, il fait écho aux annotations en marge que nous venons de voir et d'autre part il se présente comme un script de la trame qui a été développée durant les chapitres précédents. L'énonciateur s'adresse à un étranger désigné par un démonstratif qui induit la précision et que pourtant rien ne peut identifier, qui n'a aucun référent, ni textuel, ni réel :

«Oui, j'ai décidé de répondre aux questions que m'a posées cet étranger même si je ne vois pas pourquoi il recueille de tels renseignements ».

Dans ce roman tourmenté comme une sculpture du Bernin ou comme les retables des églises du Minas, « l'énonciation s'immisce dans l'énoncé » pour reprendre les termes de Dominique Maingueneau. Le tragique de la situation de Nina, dont la présence ouvre le Domaine à la parole, force le silence ancestral à se dissoudre dans le discours enchevêtré des narrateurs et l'excès illocutoire anéantit tous les repères des personnages qui n'existaient que par le secret : la maison est assassinée par le langage dans un acte de co-énonciation. 